\title{
Efeito dos movimentos sacádicos horizontal e vertical dos olhos sobre o controle postural de adultos jovens e idosos em diferentes bases de apoio
}

\author{
Gisele Chiozi GOTARDI* \\ Sérgio Tosi RODRIGUES* \\ Daniela GODOI $^{* *}$ \\ Fabio Augusto BARBIERI* \\ Martina NAVARRO ${ }^{* * * *}$ \\ Paulo SCHOR ${ }^{* * * *}$ \\ Paula Fávaro POLASTRI*
}

*Faculdade de

Ciências, Universidade

Estadual Paulista Júlio

de Mesquita Filho,

Bauru, SP, Brasil.

**Departamento de

Educação Física e

Motricidade Humana

Universidade Federal

de São Carlos,

São Carlos, SP, Brasil.

***Escola Paulista

de Medicina,

Universidade Federal

de São Paulo,

São Paulo, SP, Brasil.

\section{Resumo}

0 objetivo deste estudo foi investigar o efeito dos movimentos sacádicos, horizontal e vertical, dos olhos no desempenho do controle postural de adultos jovens e idosos durante a manutenção da postura em pé em diferentes bases de apoio. Quinze adultos jovens e quinze idosos permaneceram parados em pé, sobre uma plataforma de força, nas bases de apoio bipodal e semi tandem, durante a execução de duas tarefas visuais, fixação de um alvo e movimentos sacádicos dos olhos nas direções horizontal e vertical. 0 Centro de Pressão (CP) foi medido nas direções de movimento anteroposterior (AP) e médio-lateral (ML). Os resultados mostraram que a frequência AP e a área de deslocamento do CP foram menores durante os movimentos sacádicos do que durante a fixação do alvo somente para os adultos jovens. Os resultados também revelaram que os idosos tiveram maior velocidade e frequência $\mathrm{ML}$ de deslocamento do CP do que os adultos jovens durante a base de apoio semi tandem. Não houve efeito de direção dos movimentos sacádicos (horizontal e vertical) para adultos jovens e idosos. Os resultados sugerem que os idosos não foram capazes de realizar os ajustes posturais necessários às tarefas visuais envolvendo os movimentos sacádicos, contribuindo para uma maior instabilidade postural em comparação aos adultos jovens.

PalavaAs-Chave: Movimentos sacádicos; Fixação; Controle postural; Envelhecimento; Bases de apoio.

\section{Introdução}

A influência do controle do olhar sobre a postura vem sendo investigada através dos movimentos dos olhos. Os movimentos dos olhos podem apresentar diferentes comportamentos durante a busca visual que estão diretamente relacionados às demandas do ambiente, tais como fixação, perseguição e movimentos sacádicos ${ }^{1,2}$. Particularmente, os movimentos sacádicos são movimentos rápidos dos olhos (velocidade média $\left.500^{\circ} / \mathrm{s}\right)^{3}$ realizados para trazer uma nova parte do campo visual para região da fóvea, característica crucial quando há movimento do alvo em diferentes amplitudes ou frequências. Tem sido sugerido que o sistema de controle postural recebe além das informaçóes visuais de deslizamento das imagens projetadas na retina, informaçôes aferentes e eferentes dos músculos que movimentam os olhos para a manutenção de uma posição corporal desejada o que auxilia significativamente na redução da oscilaçáo corporal ${ }^{4}$.

A fim de investigar o efeito dos movimentos sacádicos sobre o controle postural, REY et al. ${ }^{5}$ utilizaram a movimentação de alvos visuais, 
nas direçôes horizontal e vertical, para guiar os movimentos sacádicos dos olhos de adultos jovens durante a manutenção da posiçẫo em pé sob uma base bipodal. Os resultados indicaram que a amplitude média dos movimentos sacádicos dos olhos foi similar à amplitude média da movimentação dos estímulos (horizontal e vertical). Esses autores observaram também que durante condiçóes de fixação do olhar em um alvo posicionado em distâncias de $40 \mathrm{~cm}$ e $200 \mathrm{~cm}$, houve um aumento na amplitude e velocidade média de deslocamento do Centro de Pressão (CP), na direção anteroposterior (AP) dos indivíduos em comparação às condições de movimentos sacádicos tanto horizontais quanto verticais, corroborando outros estudos que indicaram que estes movimentos oculares não deterioram a estabilidade corporal como tradicionalmente sugerido ${ }^{5,6}$.

Rodrigues et al..$^{6}$ investigaram a influência de diferentes frequências dos movimentos sacádicos horizontais sobre a estabilidade corporal de adultos jovens, acrescentando um desafio adicional ao sistema de controle postural ao posicionar os participantes em diferentes bases de apoio. Apesar de outros estudos já terem demonstrado aumento da oscilação corporal, na medida em que a tarefa postural se torna mais desafiadora ${ }^{7,8}$, poucos estudos investigaram o efeito dos movimentos sacádicos em situaçóes que exigem maior estabilidade corporal ${ }^{9,10}$. Os resultados deste estudo indicaram diminuição na velocidade, frequência e amplitude média de deslocamento do $\mathrm{CP}$ somente quando os indivíduos estavam em uma base de apoio ampla em comparação à uma base restrita o que sugere interferência da complexidade da tarefa postural sobre o comportamento do olhar. Portanto, há evidências da redução das oscilaçóes corporais de adultos jovens quando são realizadas tarefas com os movimentos sacádicos dos olhos em comparação à de fixação do olhar ${ }^{6,11-13} \mathrm{e}$, ainda, que tarefas posturais mais desafiadoras podem interferir neste comportamento, o que sugere uma forte relação entre o comportamento do olhar e da postura.

Alguns autores defendem que a atenuação da oscilação corporal seria decorrente do fato de que os movimentos sacádicos atuariam como uma tarefa suprapostural $^{9}$ na qual o indivíduo é solicitado a movimentar os olhos enquanto mantém o corpo parado em pé simultaneamente. Neste caso, a oscilaçáo corporal seria reduzida para que a tarefa visual fosse cumprida adequadamente. Outra possível explicação é a de que a informação sensorial adicional proveniente dos músculos extraoculares seria utilizada juntamente com as informaçôes de fluxo óptico e de deslizamento da imagem projetada na retina ${ }^{4}$ para o aumento da estabilidade postural durante a tarefa de movimentos sacádicos dos olhos.

É interessante notar que o controle do olhar e da postura parecem variar com a idade do indivíduo ${ }^{7,11,13}$. De maneira geral, o processo natural de envelhecimento provoca, dentre outras alteraçóes, deterioração no controle motor e na percepção visual. Idosos apresentam resultados inferiores em algumas funçôes visuais em comparação aos adultos jovens, tais como déficits no contraste ${ }^{14}$, acuidade visual ${ }^{15} \mathrm{e}$ maior latência dos movimentos sacádicos dos olhos ${ }^{16}$. Essas alterações no sistema visual podem estar associadas a diferenças no comportamento do olhar durante a busca visual podendo resultar em erros no julgamento de distância e na percepção do espaço quando o idoso está se locomovendo, tornando-o, por exemplo, mais susceptível a sofrer quedas ${ }^{17}$.

Aguiar et al. ${ }^{11}$ verificaram o efeito dos movimentos sacádicos horizontais de um alvo $(0,5 \mathrm{~Hz}$ e $1,1 \mathrm{~Hz})$ na oscilação corporal de idosos em diferentes bases de suporte (ampla e restrita) e apontaram que, assim como em adultos jovens, as oscilaçóes foram reduzidas com os movimentos sacádicos de diferentes frequências do alvo em comparação à condição de fixação do olhar. No entanto, diferentemente dos adultos jovens, os idosos não apresentaram diferenças na magnitude das oscilações corporais entre as bases de suporte. Já Kapoula e L $\hat{E}^{18}$ demonstraram que embora os idosos tenham apresentado um comportamento do olhar similar aos adultos jovens, maior variabilidade na velocidade de oscilação foi observada nestes indivíduos em diferentes condiçóes de distância de visualização do alvo $(40 \mathrm{~cm}$ e $200 \mathrm{~cm}$ ) e dificuldade da tarefa visual (fixação x sacadas verticais). Ainda, YANG e KAPOULA ${ }^{16}$ mostraram que a latência entre a apresentação do estímulo visual e o início dos movimentos sacádicos verticais aumentam com o envelhecimento sugerindo degeneração de áreas corticais responsáveis pelo início destes movimentos. Em suma, os movimentos sacádicos dos olhos parecem reduzir as oscilaçóes corporais de adultos jovens, entretanto, a relação postura-olhar se mostra alterada e mais variável em idosos. Sendo assim, a relaçáo entre o comportamento do olhar envolvendo os movimentos sacádicos e as oscilaçôes corporais de idosos ainda é uma questáo a ser investigada ${ }^{9,11,18}$.

Ainda não está claro, por exemplo, se as direções dos movimentos sacádicos dos olhos (horizontal e vertical) e o aumento da dificuldade da tarefa por meio de redução da base de apoio influenciariam o 
comportamento do olhar e da postura de idosos assim como fazem em adultos. Estes questionamentos se apoiam no fato de que estudos têm mostrado que o rastreamento ocular horizontal é mais preciso do que o vertical tanto para bebês de cinco, sete e nove meses de idade ${ }^{19}$ quanto para adultos ${ }^{20}$ já que a maioria dos objetos rastreados no ambiente estão no plano horizontal ${ }^{21}$. Será que a direção dos movimentos dos olhos poderia alterar diferentemente as respostas posturais de idosos? Ainda, será que aumento da instabilidade postural provocada pelas bases de apoio em diferentes direçóes de movimento corporal (AP e médio-lateral - ML) poderia afetar a relaçáo olhar-postura em adultos jovens e idosos? Por fim, estas alteraçóes seriam dependentes da direção dos movimentos sacádicos (horizontais e verticais)? LAUFER et al. ${ }^{7}$ observaram que idosos aumentaram a frequência de deslocamento do CP quando a superfície de apoio foi elevada do cháo (tarefa desafiadora), tanto com os olhos abertos quanto fechados, indicando que o sistema de controle postural dos idosos foi mais exigido com o aumento da complexidade da tarefa em comparação aos adultos jovens. Os autores sugeriram que idosos tendem a ter respostas posturais mais exageradas em comparaçâo aos adultos jovens em situaçóes que possam prejudicar sua estabilidade postural, indicando a adoção de uma estratégia

\section{Método}

\section{Participantes}

Participaram desse estudo quinze idosos ativos $(71,93 \pm 5,98$ anos) e quinze adultos jovens ativos $(22,80 \pm 3,17$ anos) sem qualquer comprometimento neurológico, musculoesquelético e, quando necessário, com visão corrigida. Os participantes assinaram o Termo de Consentimento Livre e Esclarecido, aprovado pelo Comitê de Ética local (processo $n^{\circ} 11322 / 46 / 01 / 12$ - aprovação 22/11/2012).

\section{Procedimentos}

Os participantes permaneceram em pé sobre uma plataforma de força (AMTI - AccuGait), e realizaram três tarefas visuais que consistiam em fixar o olhar em um alvo projetado (multimedia BenQ-MX720) em uma tela de projeção Projetelas (modelo Infinity), $204 \mathrm{~cm}$ x $154 \mathrm{~cm}$, posicionada à frente do participante, de enrijecimento do tornozelo pouco adequada às demandas do ambiente e da tarefa ${ }^{7}$.

O presente estudo é pioneiro em investigar movimentos sacádicos horizontal e vertical durante a manutenção da estabilidade postural de adultos jovens e idosos em diferentes bases de apoio. O entendimento de como se dá o comportamento do olhar na busca por informações visuais presentes no ambiente e qual a influência deste comportamento nas respostas posturais pode contribuir consideravelmente para a elaboração de programas de atividade física que visem a melhora da qualidade de vida do idoso e prevenção de riscos de quedas associados às alteraçóes decorrentes do processo de envelhecimento.

Portanto, o objetivo do presente estudo foi investigar os efeitos de movimentos sacádicos horizontal e vertical dos olhos no desempenho do controle postural de adultos jovens e idosos durante a manutenção da posição em pé em diferentes bases de apoio. As hipóteses foram: a) movimentos sacádicos dos olhos reduzem as oscilaçóes corporais de idosos e adultos jovens; b) as direçóes horizontal e vertical dos movimentos sacádicos produzem efeitos distintos sobre as oscilaçóes corporais de idosos e adultos jovens; e c) os movimentos sacádicos têm menor influência na oscilação corporal durante a manutenção da postura em base de apoio mais desafiadora.

com o centro da tela à altura dos seus olhos, à distância de $400 \mathrm{~cm}$. O alvo consistiu em um círculo vermelho de aproximadamente $1,15^{\circ}$ de diâmetro que direcionou o olhar para as seguintes condiçóes: a) fixação do alvo (FX) - o alvo permaneceu estacionário no centro da tela; b) movimentos sacádicos horizontais do alvo $(\mathrm{SH})$ - o alvo foi apresentado para o lado direito e para o lado esquerdo, sucessivamente e c) movimentos sacádicos verticais do alvo (SV) - o alvo foi apresentado para cima e para baixo sucessivamente. $\mathrm{Na}$ tarefa de movimentos sacádicos, os participantes deveriam realizar movimentos sacádicos dos olhos de acordo com as características de amplitude e direção do alvo sendo este apresentado em uma frequência de $1,1 \mathrm{~Hz}$ e permaneceu com uma amplitude constante de $5,5^{\circ}$ do centro da tela totalizando $11^{\circ}$ tanto na direção horizontal quanto na direção vertical a fim de evitar movimentos da cabeça durante a realizaçáo da tarefa visual. 
Enquanto realizavam a tarefa visual, os participantes permaneceram sob duas condições de base de apoio: a) base bipodal (BP) - pés paralelos e alinhados aproximadamente à largura dos ombros $\mathrm{e}$ b) base semi tandem (ST) - pé dominante alinhado à frente e medialmente ao pé não dominante. Cada participante realizou dois blocos com duas tentativas em cada condição, totalizando 12 tentativas com duraçáo de 40 segundos cada. $\mathrm{O}$ intervalo entre os blocos foi de um minuto para evitar fadiga. A ordem das condiçóes foi randomizada para evitar efeito de ordem.

\section{Análise de dados e análise estatística}

Para mensurar a oscilação corporal dos participantes nas direçóes de movimento corporal, AP e ML, as informaçóes dos componentes tridimensionais da força de reação do solo (Fx, Fy e Fz) e os momentos destas forças ( $\mathrm{Mx}, \mathrm{My}$ e $\mathrm{Mz}$ ) provenientes dos sensores (strain ganges) localizados nas extremidades da plataforma de força foram utilizados para cálculo do deslocamento do Centro de Pressão $(\mathrm{CP})^{22}$, com frequência de amostragem de $60 \mathrm{~Hz}$. Para avaliar os efeitos de movimentos sacádicos horizontal e vertical e diferentes bases de apoio sobre o deslocamento do CP foram calculadas as seguintes variáveis dependentes: velocidade média e frequência mediana de deslocamento do $\mathrm{CP}$, nas direçôes AP e ML e área de deslocamento do CP.

\section{Resultados e Discussão}

Para a velocidade média de deslocamento do CP (FIGURA 1), MANOVA apontou diferenças para o fator grupo, Wilk's Lambda $=0,528$, $\mathrm{F}(2,27)=12,074, \mathrm{p}<0,0001$, para o fator base, Wilk's Lambda $=0,069, F(2,27)=182,427, p<0,0001$, interação entre grupo e base, Wilk's Lambda $=0,540$, $\mathrm{F}(2,27)=11,482, \mathrm{p}<0,0001$ e interação entre grupo, base e tarefas visuais, Wilk's Lambda $=0,628$, $\mathrm{F}(4,25)=3,699, \mathrm{p}<0,05$. Análises univariadas revelaram que para o fator grupo, a diferença foi tanto na direção $\mathrm{AP}, \mathrm{F}(1,28)=16,404, \mathrm{p}<0,0001$, quanto na direção $\mathrm{ML}, \mathrm{F}(1,28)=24,906, \mathrm{p}<0,0001$, indicando que os idosos tiveram maior velocidade de deslocamento do CP (AP: $M=1,216 \mathrm{~cm} / \mathrm{s}$, $\mathrm{DP}=0,07$ e $\mathrm{ML}: \mathrm{M}=1,391 \mathrm{~cm} / \mathrm{s}, \mathrm{DP}=0,08)$ do que os adultos jovens (AP: $\mathrm{M}=0,814 \mathrm{~cm} / \mathrm{s}$, $\mathrm{DP}=0,07$ e ML: $\mathrm{M}=0,836 \mathrm{~cm} / \mathrm{s}, \mathrm{DP}=0,08)$. Para o fator base, a diferença foi tanto para a direção
A variável velocidade média foi calculada a partir das trajetórias de deslocamento do CP divididas pelo tempo total da tentativa. A variável frequência mediana foi computada pela estimativa de densidade espectral (PSD) utilizando-se o Método Welch, fixado em segmento de 1.024 pontos, com sobreposição de $50 \%$. A variável área de deslocamento do $\mathrm{CP}$ foi computada a partir do cálculo de uma elipse que englobou $85 \%$ dos dados de oscilação corporal; os dois eixos desta elipse foram calculados a partir das medidas de dispersão dos sinais por meio do método de Análise dos Componentes Principais. Os dados provenientes da plataforma de força foram carregados e analisados, por um grupo de rotinas específicas escritas na linguagem MATLAB (10.0 MathWorks, Inc). Os dados foram filtrados por um filtro Butterworth (passa-baixa, segunda ordem) com frequência de corte de $4 \mathrm{~Hz}$.

Foram realizadas duas análises de multivariância (MANOVA) e uma análise de variância (ANOVA) tendo como fatores: grupo (adultos jovens e idosos), base de apoio (BP e ST) e tarefas visuais (FX, SH, $\mathrm{SV})$ sendo os fatores Base de apoio e Tarefas visuais considerados como medidas repetidas. As variáveis dependentes para as MANOVAs foram a velocidade média e a frequência mediana de deslocamento do $\mathrm{CP}$ - AP e ML, e para a ANOVA foi a área de deslocamento do CP. Quando necessário, foi utilizado teste de post hoc de Bonferroni. O nível de significância adotado para todas as análises foi de 0,05 (SPSS 17.0).

AP, $\mathrm{F}(1,28)=116,868, \mathrm{p}<0,0001$, quanto para a direçáo ML, $\mathrm{F}(1,28)=364,893$, $\mathrm{p}<0,0001$, indicando que na base de apoio ST (AP: $M=1,237 \mathrm{~cm} / \mathrm{s}$, $\mathrm{DP}=0,055$ e ML: $\mathrm{M}=1,666 \mathrm{~cm} / \mathrm{s}, \mathrm{DP}=0,07)$ os participantes apresentaram maior velocidade de deslocamento do $\mathrm{CP}$ em comparação à base de apoio BP (AP: $\mathrm{M}=0,793 \mathrm{~cm} / \mathrm{s}, \mathrm{DP}=0,05$ e ML: $\mathrm{M}=0,561 \mathrm{~cm} / \mathrm{s}, \mathrm{DP}=0,05)$. A interação entre grupo e base foi observada apenas para a direção ML, $\mathrm{F}(1,28)=22,170, \mathrm{p}<0,0001$, indicando que o aumento da velocidade de deslocamento do $\mathrm{CP}$ dos idosos $(\mathrm{M}=2,080 \mathrm{~cm} / \mathrm{s}, \mathrm{DP}=0,107)$ em relação aos adultos $(\mathrm{M}=1,252 \mathrm{~cm} / \mathrm{s}, \mathrm{DP}=0,11)$ foi ainda maior quando os participantes estavam na base de apoio ST $(\mathrm{M}=0,70 \mathrm{~cm} / \mathrm{s}, \mathrm{DP}=0,66)$ do que na base de apoio $\mathrm{BP}(\mathrm{M}=0,42 \mathrm{~cm} / \mathrm{s}, \mathrm{DP}=0,66)$. Para a interação grupo, base e tarefa visual, as análises univariadas não apontaram diferenças ( $\mathrm{p}>0.05)$. 

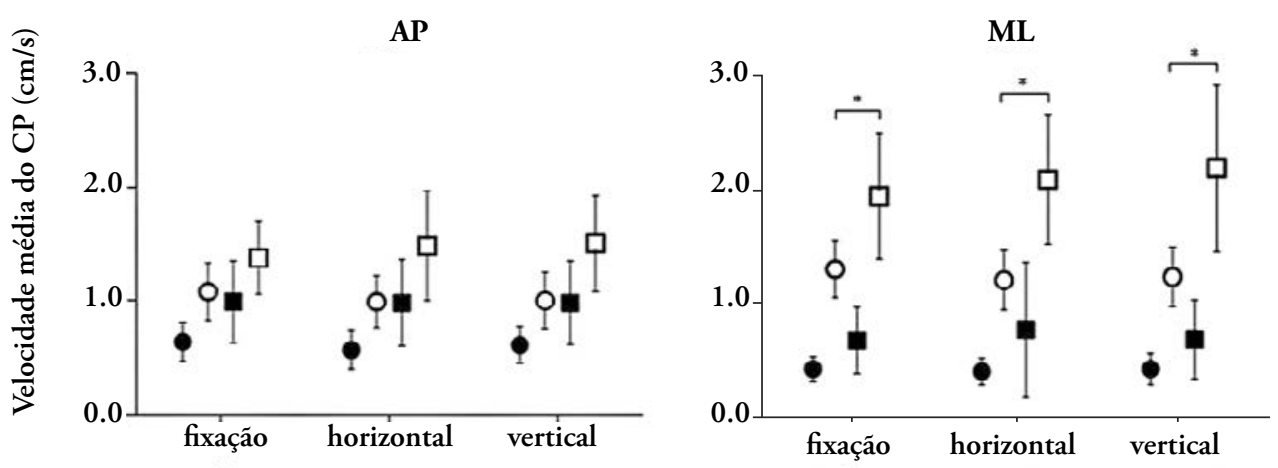

* interação entre os
fatores base de apoio
e grupo.

FIGURA 1 - Velocidade média de deslocamento do Centro de Pressão (CP) nas direções anteroposterior (AP) e médio-lateral (ML), para os dois grupos experimentais (adultos e idosos) nas três tarefas visuais (fixação, horizontal e vertical) e duas bases de apoio (bipodal - bp e semi tandem - st).

Para a frequência mediana de deslocamento do CP (FIGURA 2), MANOVA revelou diferenças para o fator grupo, Wilk's Lambda $=0,673, \mathrm{~F}(2,27)=6,545$, $\mathrm{p}<0,005$, para o fator base, Wilk's Lambda $=0,672$, $\mathrm{F}(2,27)=6,577, \mathrm{p}<0,005$, interação entre grupo e base, Wilk's Lambda $=0,657, F(2,27)=7,036$, $\mathrm{p}<0,003$ e interação entre grupo e tarefas visuais, Wilk's Lambda $=0,616, \mathrm{~F}(4,25)=3,889, \mathrm{p}<0,02$. Análises univariadas apontaram que, para o fator grupo, a diferença foi tanto na direção $\mathrm{AP}, \mathrm{F}(1,28)=12,136$, $\mathrm{p}<0,002$, quanto na $\mathrm{ML}, \mathrm{F}(1,28)=6,301, \mathrm{p}<0,02$, evidenciando que os idosos tiveram maior frequência mediana de deslocamento (AP: $M=0,26 \mathrm{~Hz}$, $\mathrm{DP}=0,02$ e ML: $\mathrm{M}=0,27 \mathrm{~Hz}, \mathrm{DP}=0,016)$ do que os adultos jovens (AP: $\mathrm{M}=0,16 \mathrm{~Hz}, \mathrm{DP}=0,02 \mathrm{e}$ ML: $M=0,27 \mathrm{~Hz}, \mathrm{DP}=0,016)$. Para o fator base, as diferenças foram encontradas somente para a direção AP, $F(1,28)=13,573, p<0,001$. Ambos os grupos tiveram maior frequência mediana de deslocamento do CP na base de apoio ST $(M=0,25 \mathrm{~Hz}, \mathrm{DP}=0,012)$ do que na base de apoio $\mathrm{BP}(\mathrm{M}=0,16 \mathrm{~Hz}$, $\mathrm{DP}=0,023)$. A interação entre grupo e base foi observada somente na direção $\mathrm{ML}, \mathrm{F}(1,28)=14,425$, $\mathrm{p}<0,001$. Testes post hoc mostraram que os adultos $(\mathrm{M}=0,16 \mathrm{~Hz}, \mathrm{DP}=0,017)$ apresentaram menor frequência mediana de deslocamento do $\mathrm{CP}$ em comparação aos idosos $(\mathrm{M}=0,29 \mathrm{~Hz}, \mathrm{DP}=0,017)$ somente na base de apoio ST. A interaçáo entre grupo e tarefas visuais foi significativa somente para a direção $\mathrm{AP}, \mathrm{F}(2,56)=8,535, \mathrm{p}<0,001$. Testes post hoc mostraram que os adultos apresentaram menor frequência mediana de deslocamento do $\mathrm{CP}$ em todas as tarefas visuais quando comparados aos idosos. Interessantemente, somente os adultos jovens apresentaram menor frequência mediana durante a tarefa $\mathrm{FX}(\mathrm{M}=0,13 \mathrm{~Hz}, \mathrm{DP}=0,023)$, em comparação as tarefas $\mathrm{SH}(\mathrm{M}=0,16 \mathrm{~Hz}, \mathrm{DP}=0,022)$ e $S V(M=0,17 \mathrm{~Hz}, \mathrm{DP}=0,023)$, enquanto que os idosos apresentaram frequência mediana menor nas tarefas $\mathrm{SH}(\mathrm{M}=0,24 \mathrm{~Hz}, \mathrm{DP}=0,022) \mathrm{e}$ SV $(M=0,24 \mathrm{~Hz}, \mathrm{DP}=0,023)$ em comparação à tarefa $\mathrm{FX}(\mathrm{M}=0,28 \mathrm{~Hz}, \mathrm{DP}=0,023)$.

Para a área de deslocamento do CP (FIGURA 3), ANOVA revelou diferenças para o fator grupo, $\mathrm{F}(1,28)=5,637, \mathrm{p}<0,05$, para o fator base $F(1,28)=81,508, p<0,0001$, e interaçáo entre grupo e tarefas visuais, $\mathrm{F}(2,56)=5,335, \mathrm{p}<0,01$. Os idosos apresentaram maior área de deslocamento do $\mathrm{CP}\left(\mathrm{M}=3,272 \mathrm{~cm}^{2}, \mathrm{DP}=0,309\right)$ em comparação aos adultos jovens $\left(\mathrm{M}=2,236 \mathrm{~cm}^{2}, \mathrm{DP}=0,309\right) \mathrm{em}$ todas as condições experimentais, e ambos os grupos mostraram maior área de deslocamento do CP quando estavam na base de apoio $S T\left(M=3,775 \mathrm{~cm}^{2}\right.$, $\mathrm{DP}=0,30)$ em comparação a base de apoio BP $\left(\mathrm{M}=1,733 \mathrm{~cm}^{2}, \mathrm{DP}=0,175\right)$. Na interação grupo e tarefa visual, testes post hoc apontaram que os idosos (horizontal: $\mathrm{M}=3,251 \mathrm{~cm}^{2}, \mathrm{DP}=0,307$, vertical: $\left.\mathrm{M}=3,448 \mathrm{~cm}^{2}, \mathrm{DP}=0,369\right)$ oscilaram mais do que os adultos jovens (horizontal: $\mathrm{M}=1,950 \mathrm{~cm}^{2}$, $\mathrm{DP}=0,307$, vertical: $\mathrm{M}=2,063 \mathrm{~cm}^{2}, \mathrm{DP}=0,369$ ) durante a realização das tarefas $\mathrm{SH}$ e $\mathrm{SV}$, mas não durante a tarefa FX (idosos: $M=3,118 \mathrm{~cm}^{2}$, $\mathrm{DP}=0,329$, adultos: $\mathrm{M}=2,694 \mathrm{~cm}^{2}, \mathrm{DP}=0,329$ ). Ainda, somente o grupo dos adultos jovens reduziu a área de deslocamento do CP durante as tarefas $\mathrm{SH}$ e $\mathrm{SV}$. 
* interação entre os fatores base de apoio e grupo e + interação entre os fatores tarefa visual e grupo.
+ interação entre os fatores tarefa visual e grupo.
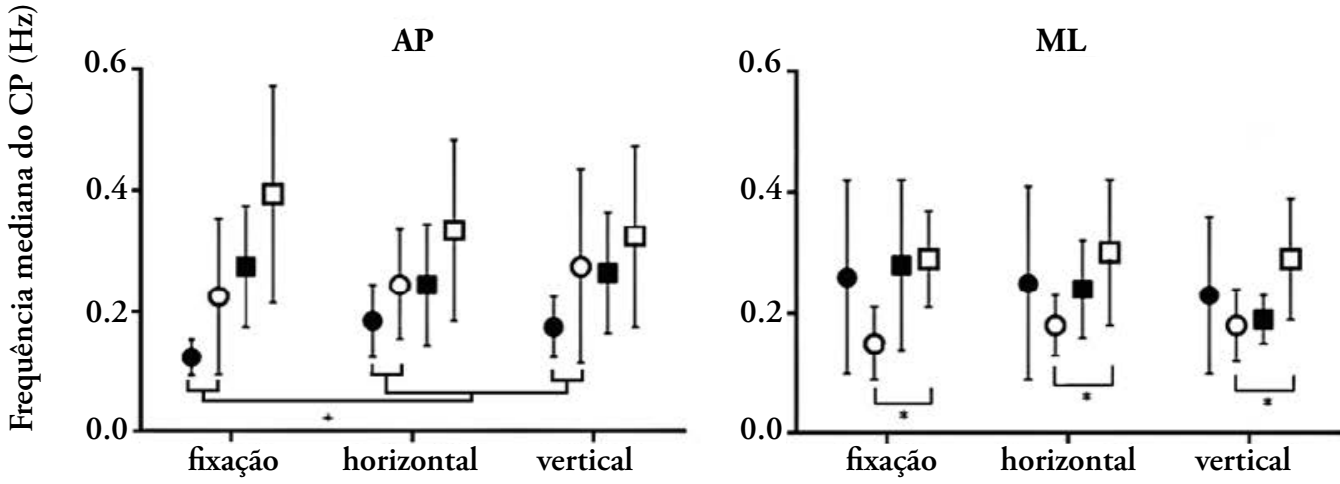

FIGURA 2 - Frequência mediana de deslocamento do Centro de Pressão (CP) nas direções anteroposterior (AP) e médio-lateral (ML), para os dois grupos experimentais (adultos e idosos) nas três tarefas visuais (fixação, horizontal e vertical) e duas bases de apoio (bipodal - bp e semi tandem - st).

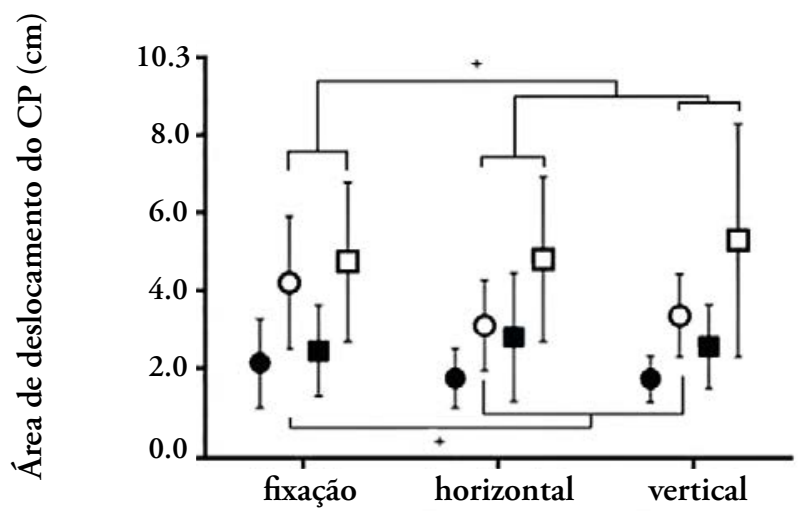

FIGURA 3 - Área de deslocamento do Centro de Pressão (CP) para os dois grupos experimentais (adultos e idosos) nas três tarefas visuais (fixação, horizontal e vertical) e duas bases de apoio (bipodal - bp e semi tandem - st).

Os resultados confirmam que os movimentos sacádicos dos olhos, guiados por meio de um alvo visual, reduzem as oscilaçôes corporais de adultos jovens ${ }^{5,6,9,11,12}$. No entanto, a hipótese de que idosos reduziriam o deslocamento do $\mathrm{CP}$ durante a tarefa de movimentos sacádicos não foi confirmada, em desacordo com achados de Aguiar et al. ${ }^{11}$ que revelaram uma magnitude e velocidade de deslocamento do CP similar de adultos jovens e idosos em todas as condiçóes do olhar, independentemente da dificuldade da tarefa postural, além de tendência oposta na frequência mediana de deslocamento do CP. Estas contradições podem estar relacionadas às diferenças metodológicas entre os estudos, sendo que Aguiar et al. ${ }^{11}$ utilizou a análise de oscilação do tronco e cabeça enquanto o presente estudo analisou o deslocamento do CP. É interessante notar que os resultados de Aguiar et al. ${ }^{11}$ corroboram os de STOFFregen et al. ${ }^{9}$ que indicaram redução nas oscilações da cabeça mas náo no deslocamento do $\mathrm{CP}$ o que sugere que os idosos apresentaram comportamentos diferentes entre a oscilação dos segmentos corporais e o deslocamento do CP para atenuaçáo da estabilidade postural durante as tarefas propostas haja vista que, nestas condiçóes, ambos os métodos de análise do 
controle postural têm indicado diferenças similares nas respostas posturais ${ }^{8,22,23}$.

Uma possível explicação é que o presente estudo propôs uma distância maior de visualizaçáo do alvo $(400 \mathrm{~cm})$ o que pode ter influenciado estes resultados. KaPOULA e L $\hat{E}^{18}$ indicaram que adultos jovens e idosos oscilaram mais durante a condição de distância de visualização de $200 \mathrm{~cm}$ do que durante a distância de $40 \mathrm{~cm}$ ao realizarem movimentos sacádicos verticais e fixação do alvo, sugerindo que este resultado estava associado ao tamanho angular do deslizamento da imagem na retina: quanto mais longe do alvo, menor o ângulo de deslizamento da imagem na retina, dificultando o reconhecimento do deslocamento visual quando o corpo está oscilando. Somente com a distância de visualização de $40 \mathrm{~cm}$, ambos os grupos reduziram as oscilaçôes corporais durante os movimentos sacádicos verticais do alvo, se comparados à fixação.

$\mathrm{O}$ efeito dos movimentos sacádicos foi indicado pela menor frequência mediana e magnitude de deslocamento do CP de adultos jovens, o que não foi observado nos idosos. Freitas e Duarte ${ }^{23}$ indicaram que quanto menores as frequências de oscilação corporal, menores as diferenças entre as respostas neuromusculares, representadas pelo deslocamento do centro de pressão, e a oscilação corporal, representada pelo deslocamento do centro de gravidade. Pode-se notar que adultos jovens apresentaram frequências medianas de oscilação corporal menores do que idosos em todas as condiçôes experimentais (direção AP) e menores durante a tarefa de FX indicando melhor controle corporal sobre as alteraçóes impostas pelas tarefas visuais e posturais. Por outro lado, os idosos apresentaram diminuição da frequência mediana durante as tarefas $\mathrm{SH}$ e SV o que demonstra a tentativa de melhor estabilização corporal para cumprir as tarefas impostas. Embora a distância entre a tela e o olho do participante possa ser compreendida como uma dificuldade adicional da tarefa para ambos os grupos, os idosos foram os mais afetados por ela, sendo que não conseguiram reduzir suas respostas posturais durante a realização dos movimentos sacádicos, como em estudo prévio ${ }^{18}$. A maior frequência mediana observada para os idosos em todas as condiçóes parece evidenciar a maior instabilidade durante a manutenção da posição em pé.

Os resultados sugerem que a relaçáo entre o controle do olhar e da postura é alterada em idosos. STOFFrEgEn et al. ${ }^{9,10}$ têm proposto que as oscilaçôes corporais são minimizadas para facilitar a execução da tarefa suprapostural, neste caso, os movimentos sacádicos. Stoffregen et al. ${ }^{10}$ demonstraram que durante os movimentos sacádicos guiados por um estímulo visual, os adultos oscilaram menos do que durante os movimentos sacádicos com os olhos fechados e a fixação do olhar. Os autores atribuíram estes resultados a um controle postural facilitatório, no qual o principal objetivo do sistema de controle postural não foi minimizar as oscilaçóes corporais per se, mas sim facilitar o desempenho da tarefa. Sendo assim, se a tarefa suprapostural exige alta precisão, as oscilaçôes corporais são minimizadas para que o desempenho da tarefa não seja prejudicado.

$\mathrm{O}$ controle postural facilitatório ${ }^{10}$ tem sido utilizado para contra argumentar a hipótese de que durante a execução de uma tarefa dual ocorre uma competição central de recursos, implicando em uma deterioração na performance da tarefa postural ${ }^{24}$. Essa hipótese ${ }^{24}$ tem sido interpretada como referente a um controle postural autônomo, no qual o primeiro objetivo é minimizar as oscilaçóes corporais para manter a estabilidade postural e as informaçóes da tarefa suprapostural tornam-se concorrentes com as informaçóes que mantêm a estabilidade postural. No entanto, Mitra e Fraizer ${ }^{25}$ sugerem que, ao invés de um sistema que compete entre recursos, pode existir um compartilhamento adaptativo de recursos que contribui para a execução da tarefa dual. Ora o controle postural pode ser facilitatório ora autônomo, dependendo das demandas das tarefas postural e suprapostural. Assim, o sistema é capaz de compartilhar ou priorizar entre os componentes, postural e suprapostural. No presente estudo, os idosos mantiveram a mesma área e frequência mediana de deslocamento do CP quando realizaram a fixação e os movimentos sacádicos nas duas bases de apoio. Neste caso, a precisão da tarefa suprapostural (fixar o centro do círculo) e a dificuldade da tarefa postural (manter-se estável visualizando um alvo à $400 \mathrm{~cm}$ ) representaram para os idosos uma demanda alta de dificuldade da tarefa. Utilizando a ideia de compartilhamento adaptativo dos recursos proposto por Mitra e Fraizer ${ }^{25}$, pode-se observar não somente os efeitos da tarefa suprapostural na oscilação corporal, mas também os efeitos da dificuldade da tarefa postural no desempenho da tarefa suprapostural.

A hipótese de que diferentes direçôes dos movimentos sacádicos (horizontais e verticais) alterariam a oscilaçáo corporal de adultos jovens e idosos também não foi confirmada. Estudos ${ }^{19,20}$ têm apontado que o desenvolvimento do controle do olhar na direçáo horizontal ocorre antes do que na direçâo vertical. Uma possível explicação 
para a assimetria entre os movimentos dos olhos horizontais e verticais seria o fato da maioria dos objetos rastreados pelos olhos no ambiente estarem dispostos no plano horizontal e, assim, maior número de movimentos dos olhos ser realizado na direção horizontal ${ }^{21}$. Outras evidências ${ }^{5,19}$ indicaram ainda que com o passar dos anos e, mesmo com o processo de envelhecimento, alteraçóes na direção do olhar não influenciam a manutenção da postura em pé. No entanto, a hipótese sobre a direção do olhar foi baseada no fato de que os movimentos sacádicos poderiam auxiliar na redução da instabilidade gerada pela base de apoio e que esta redução seria dependente da direção do olhar. Idosos permanecendo em uma base de apoio instável poderiam se beneficiar reduzindo as oscilaçóes corporais ao realizar os movimentos sacádicos em uma determinada direçáo (horizontal ou vertical). Sendo assim, movimentos sacádicos horizontais do estímulo poderiam reduzir as oscilaçôes corporais observadas na direção de movimento corporal ML, na base de apoio semi tandem, enquanto movimentos sacádicos verticais do estímulo provocariam este efeito na direção de movimento corporal $\mathrm{AP}$, na base de apoio bipodal. Contudo, a direção do olhar não gerou alteraçôes na estabilidade dos participantes, sendo que os efeitos do envelhecimento foram mais evidentes na realização das tarefas visuais (fixação e movimentos sacádicos) e em relação à base de apoio.

Particularmente, com relaçáo à base de apoio, ambos os grupos apresentaram maior oscilação corporal durante a manutenção da postura na base de apoio ST, sendo que este aumento foi mais acentuado para os idosos somente na direção de movimento ML. Este resultado confirma que durante a base de apoio ST os participantes ficaram mais instáveis na direção $\mathrm{ML}$, e que os idosos foram mais desestabilizados do que os adultos ao assumir essa posição. Corroborando estes resultados, RoDrigues et al. ${ }^{6}$ observaram que adultos jovens aumentaram a oscilação corporal durante a base de apoio reduzida (pés alinhados e encostados) em comparação à base de apoio ampla (pés alinhados na largura dos ombros). Toledo e Barela ${ }^{26} \mathrm{e}$ Freitas Júnior e Barela ${ }^{27}$ demonstraram que os idosos apresentam declínios tanto nos sistemas sensoriais quanto motor, deteriorando o desempenho do controle postural. Estes autores sugeriram que o envelhecimento poderia causar uma diminuição na velocidade de transmissão do impulso nervoso e uma diminuição na capacidade de integração das informaçôes sensoriais o que, consequentemente, levaria ao aumento das oscilaçóes corporais.

Além de alteraçóes nas respostas posturais, PAQUETE e FunG ${ }^{28}$ demostraram alteraçóes no controle do olhar dos idosos durante perturbaçóes provocadas pelo movimento da base de suporte. Em especial, estes autores mostraram que durante a realização de movimentos sacádicos horizontais e posicionados sobre uma superfície móvel, os idosos cometeram mais erros sacádicos e apresentaram maior latência no movimento do olho em comparação aos adultos jovens. Com a superfície parada, os resultados foram similares para ambos os grupos. Os autores sugeriram que em decorrência das alteraçóes provocadas pelo processo de envelhecimento, os idosos enfrentam uma dificuldade maior em correlacionar o controle do olhar e da postura perante uma situação mais desafiadora.

No presente estudo, os idosos não apresentaram redução do deslocamento do $\mathrm{CP}$ em decorrência dos movimentos sacádicos ou de suas diferentes direçóes (horizontal e vertical) sendo que oscilaram mais do que adultos jovens e está oscilação foi ainda maior quando estavam na base de apoio ST independente das tarefas visuais. Uma das possíveis razóes para estes resultados pode estar associada à distância entre o participante e o alvo o que corrobora outros estudos que verificaram o efeito da distância do alvo sobre as oscilaçóes corporais de adultos jovens e idosos, inclusive sobre o comportamento do olhar ${ }^{5,18}$. Tendo em vista que Rodrigues et al. ${ }^{6} \mathrm{e}$ Aguiar et al. ${ }^{11}$ também manipularam as bases de suporte e tarefas visuais e mostraram efeito dos movimentos sacádicos sobre as oscilaçóes corporais de adultos jovens e idosos estando na distância de um metro do alvo, os resultados do presente estudo sugerem que a distância do alvo pode ter acrescentado uma demanda maior aos idosos para a manutenção da postura ereta tornando-os mais instáveis e incapazes de reduzir as oscilações corporais e realizar os ajustes posturais adequados às demandas das tarefas visuais.

Conclui-se que os movimentos sacádicos dos olhos não reduzem as oscilaçôes corporais de idosos como fazem em adultos jovens. Diferentes direçôes dos movimentos sacádicos não provocam alteraçôes nas respostas posturais e nem estão associadas ao aumento da dificuldade da tarefa postural com a alteração da base de apoio. Sugere-se que a capacidade de executar ajustes posturais necessários à tarefa visual é reduzida no idoso, contribuindo para um controle postural mais instável. 


\section{Abstract}

Effect of horizontal and vertical saccadic eye movements on postural control of young and old adults in different bases of support

The aim of this study was to investigate the effect of horizontal and vertical saccadic eye movements on postural control of young and older adults during the maintenance of upright stance in different bases of support. Fifteen young adults and fifteen elderly were asked to stand upright on a force platform in both bipodal and semi tandem bases of support, performing two visual tasks, fixation and horizontal and vertical saccadic eye movements. Center of Pressure (CoP) in the anterior/posterior (AP) and medial/lateral (ML) directions of movement were measured throughout trials. The results showed AP median sway frequency and magnitude of displacement of $\mathrm{CoP}$ decreased during saccadic eye movements than during gaze fixation, only to young adults. Furthermore, elderly showed higher ML median sway frequency and mean velocity of displacement of CoP than young adults during semi tandem basis of support. There was no effect of direction of saccadic eye movements (horizontal and vertical) in young and old adults. The results suggest that older adults were not able to perform necessary postural adjustments during visual tasks involving the saccadic eye movements, increasing postural instability as compared to young adults.

KEYWORDS: Saccadic eye movements; Fixation; Postural control; Elderly; Bases of support.

\section{Referências}

1. Rodrigues ST. O movimento dos olhos e a relação percepção-ação. In: Teixeira LA, editor. Avanços em comportamento motor. Rio Claro: Movimento; 2001. p. 122-46.

2. Land MF. Eye movements and the control of actions in everyday life. Prog Retin Eye Res. 2006;25(3):296-324.

3. Leigh RJ, Zee DS. The saccadic system. In: Leigh RJ, Zee DS, editors. The neurology of eye movements. 5th ed. New York: Oxford University Press; 2006. p. 79-132.

4. Guerraz M, Bronstein AM. Ocular versus extraocular control of posture and equilibrium. Clin Neurophysiol [Internet]. 2008 [citado 4 fev 2016];38(6):391-8. Disponível em: http://linkinghub.elsevier.com/retrieve/pii/S0987705308001342

5. Rey F, Lê TT, Bertin R, Kapoula Z. Saccades horizontal or vertical at near or at far do not deteriorate postural control. Auris Nasus Larynx. 2008;35(2):185-91.

6. Rodrigues ST, Aguiar SA, Polastri PF, Godoi D, Moraes R, Barela JA. Effects of saccadic eye movements on postural control stabilization. Motriz. 2013;19(3):614-9.

7. Laufer Y, Barak Y, Chemel I. Age-related differences in the effect of a perceived threat to stability on postural control. J Gerontol A Biol Sci Med Sci. 2006;61(5):500-4.

8. Barela AMF, Alveno D, Garcia C, Pereira CA. Comparação de dois métodos de análise do controle postural durante a manutenção da postura ereta e quieta. Brazilian J Mot Behav. 2009;4(1):30-6.

9. Stoffregen TA, Bardy BG, Bonnet CT, Hove P, Oullier O. Postural sway and the frequency of horizontal eye movements. Motor Control. 2007;11(1):86-102.

10. Stoffregen TA, Bardy BG, Bonnet CT, Pagulayan RJ. Postural stabilization of visually guided eye movements. Ecol Psychol. 2006;18(3):191-222.

11. Aguiar SA, Polastri PF, Godoi D, Moraes R, Barela JA, Rodrigues ST. Effects of saccadic eye movements on postural control in older adults. Psychol Neurosci. 2015;8(1):19-27.

12. Uchida T, Hashimoto M, Suzuki N, Takegami T, Iwase Y. Effects of periodic saccades on the body sway in humans subjects. Neurosci Lett. 1979;13(3):253-8.

13. Prioli AC, Freitas Junior PB, Barela JA. Physical activity and postural control in the elderly: coupling between visual information and body sway. Gerontol [Internet]. 2005 [acesso 4 fev 2016];51(3):145-8. Disponível em: http://www.karger.com/doi/10.1159/000083984

14. Owsley C. Aging and vision. Vision Res. 2011;51(13):1610-22. 
15. West SK, Munoz B, Rubin GS, et al. Function and visual impairment in a population-based study of older adults. Invest Ophthalmol Vis Sci [Internet]. 1997 [acesso 4 fev 2016];38(1):72-82. Disponível em: http://www.ncbi.nlm.nih.gov/ pubmed/9008632

16. Yang Q, Kapoula Z. The control of vertical saccades in aged subjects. Exp Brain Res. 2006;171(1):67-77.

17. Sturnieks DL, St George R, Lord SR. Balance disorders in the elderly. Neurophysiol Clin [Internet]. 2008 [acesso 4 fev 2016];38(6):467-78. Disponível em: http://linkinghub.elsevier.com/retrieve/pii/S0987705308001160

18. Kapoula Z, Lê TT. Effects of distance and gaze position on postural stability in young and old subjects. Exp Brain Res [Internet]. 2006 [acesso 4 fev 2016];173(3):438-45. Disponível em: http://link.springer.com/10.1007/s00221-006-0382-1

19. Ingster-Moati I, Vaivre-Douret L, Bui Quoc E, Albuisson E, Dufier JL, Golse B. Vertical and horizontal smooth pursuit eye movements in children: a neuro-developmental study. Eur J Paediatr Neurol. 2009;13(4):362-6.

20. Grönqvist H, Gredebäck G, Hofsten CV. Developmental asymmetries between horizontal and vertical tracking. Vision Res. 2006;46(11):1754-61.

21. Collewijn H, Tamminga EP. Human smooth and saccadic eye movements during voluntary pursuit of different target motions on different backgrounds. J Physiol. 1984;351:217-50.

22. Duarte M, Freitas SMSF. Revision of posturography based on force plate for balance evaluation. Rev Bras Fisioter. 2010;14(3):183-92.

23. Freitas SMSF, Duarte M. Métodos de análise do controle postural. São Paulo: Laboratório Biofísica, Universidade São Paulo; 2006. p. 1-21.

24. Woollacott M, Shumway-Cook A. Attention and the control of posture and gait: a review of an emerging area of research. Gait Posture. 2002;16(1):1-4.

25. Mitra S, Fraizer EV. Effects of explicit sway-minimization postural-suprapostural dual-task performance. Hum Mov Sci. 2004;23(1):1-20.

26. Toledo DR, Barela JA. Age-related differences in postural control: effects of the complexity of visual manipulation and sensorimotor contribution to postural performance. Exp Brain Res. 2014;232(2):493-502.

27. Freitas Júnior P, Barela JA. Alteraçóes no funcionamento do sistema de controle postural de idosos: uso da informação visual. Rev Port Cien Desp. 2006;6(1):94-105.

28. Paquette C, Fung J. Old age affects gaze and postural coordination. Gait Posture. 2011;33(2):227-32.
ENDEREÇO
Gisele Chiozi Gotardi
Av. Eng. Edmundo Carrijo Coube, 14-01 - Vargem Limpa Recebido para publicação: 04/02/2016 1a. Revisão: 13/07/2016 17033-360 - Bauru - SP - BRASIL e-mail: gcgotardi@gmail.com 2a. Revisão: 04/11/2016 Aceito: 26/01/2017 